\title{
Comparison of the Use of Different Doses of Intrathecal Morphine Added to Levobupivacaine for Spinal Anaesthesia During Unilateral Inguinal Hernia Repair
}

\author{
Tek Taraflı Inguinal Herni Operasyonlarında Intratekal Levobupivakain ve Levobupivakaine Eklenen Farklı \\ Dozlardaki Morfin Kullanımının Karşılaştırılması
}

Gülay Kip, Mehmet Akçabay

Gazi University Medical Faculty, Department of Anaesthesiology and Reanimation, Ankara, Turkey.

\section{ABSTRACT}

Background: Spinal anaesthesia offers safe and comfortable surgery in inguinal hernia repair. Local anaesthetic and opioid combinations are gaining popularity in intrathecal anaesthesia due to many advantages both in terms of providing patient and surgical satisfaction in addition to acceptable complication rates. In this study we aimed to compare different morphine doses added to levobupivacaine for intratechal anesthesia in unilateral inguinal hernia repair.

Material and Methods Patients were randomly divided into three groups: 12 $\mathrm{mg} / 2.5 \mathrm{ml}$ levobupivacaine only (Group L), $12 \mathrm{mg}$ levobupivacaine with $50 \mu \mathrm{g}$ morphine (Group LM $\mathrm{LM}_{50}$ ), $12 \mathrm{mg}$ levobupivacaine with $100 \mu \mathrm{g}$ morphine (Group $\mathrm{LM}_{100}$ ). Then, groups were compared for vital signs, sensorial neural block levels, motor block levels, side effects and drug therapies at the post anaesthesia period. Time of sensory and motor block, first mobilization, urination and discharge were also recorded for all patients.

Results: Mean sensory block level of patients in Group $L$ at 20th minute was significantly lower than those in Group $\mathrm{LM}_{50}$ and Group $\mathrm{LM}_{100}(\mathrm{p}<0.05)$. The mean time required to achieve maximum motor block were significantly shorter in Group LM $\mathrm{L}_{50}$ and Group $\mathrm{LM}_{100}$ than that in Group L $(p=0.008$ and $p=0.001$ respectively). The duration of motor block was significantly longer in Group $\mathrm{LM}_{50}$ and Group $\mathrm{LM}_{100}$ than that in Group L $(\mathrm{p}=0.020, \mathrm{p}=0.019$, respectively). The mean time for first postoperative analgesic demand in Group L was significantly shorter than those in Group LM $\mathrm{L}_{50}$ and Group LM $\mathrm{L}_{100}$ $(p=0.001$ and $p<0.0001$ respectively).

Conclusion: We can state that combination of levobupivacaine with different morphine doses of 50 or $100 \mu \mathrm{g}$ results in increased perioperative anaesthesia and analgesia quality without any significant post-anaesthesia complication during elective unilateral inguinal hernia repair.

Key Words: Spinal anaesthesia, inguinal hernia repair, levobupivacaine, morphine

Received: 12.09 .2017

Accepted: 12.22 .2017

\section{ÖZET}

Amaç: İnguial herni onarımında spinal anestezi güvenli ve konforlu bir cerrahi sağlamaktadır. İntratekal anestezide kullanılan lokal anestezik ve opioid kombinasyonları hasta ve cerrahi memnuniyetinin yanısıra kabul edilebilir komplikasyon oranları sağladıkları için giderek popülarite kazanmaktadır. Bu çalışmada tek taraflı inguinal herni onarımı için intratekal anestezide levobupivakaine eklenen farklı morfin dozlarını karşılaştırmayı amaçladık. Materyal ve Metod: Hastalar rastgele üç gruba ayrıldılar: sadece $12 \mathrm{mg} / 2.5 \mathrm{ml}$ levobupivakain (Grup L), 12 mg levobupivakain ve $50 \mu \mathrm{g}$ morfin (Grup LM50), $12 \mathrm{mg}$ levobupivakain ve $100 \mu \mathrm{g}$ (Grup $\mathrm{LM}_{100}$ ). Daha sonra gruplar vital bugular, duysal nöral blok düzeyleri, motor blok düzeyleri yan etkiler ve post anestezi süresindeki ilaç tedavileri açısından karşılaştırıldılar. Yine tüm hastalarda duysal ve motor blok süresi, ilk mobilizasyon, idrar yapma ve taburculuk süreleri kaydedildi.

Bulgular: Grup L deki hastaların 20. Dakikadaki ortalama duysal blok düzeyleri Grup $\mathrm{LM}_{50}$ ve Grup $\mathrm{LM}_{100}$ dekilerden anlamlı olarak düşük bulundu $(p<0.05)$. Maksimum motor bloğa ulaşmadaki ortalama süre Grup $\mathrm{LM}_{50}$ ve Grup LM $\mathrm{LM}_{100}$ 'de Grup L'dekine göre anlamlı olarak kısa bulundu ( $p=0.008$ ve $\mathrm{p}=0.001$ sırasıyla). Motor blok süresi Grup $\mathrm{LM}_{50}$ ve Grup $\mathrm{LM}_{100}$ 'de Grup L'dekine göre anlamlı olarak daha uzun bulundu ( $p=0.020, p=0.019$, sırasıyla). Postoperatif analjezik ihtiyacı için geçen ortalama süre Grup L de Grup LM L $_{50}$ ve Grup $L_{100}$ 'dekine göre anlamlı olarak daha kısa bulundu ( $p=0.001$ ve p<0.0001 sırasıyla)

Sonuç: Elektif tek taraflı inguinal herni onarımı sırasında levobupivakain ile kombine edilen 50 veya $100 \mu \mathrm{g}$ dozlarındaki morfinin herhangi bir belirgin postanestezik komplikasyona neden olmadan artmış perioperatif anestezi ve analjezi kalitesi sağladığını söyleyebiliriz.

Anahtar Sözcükler: Spinal anestezi, inguinal herni onarımı, levobupivakain, morfin

Geliş Tarihi: 09.12.2017

Kabul Tarihi: 22.12.2017 


\section{INTRODUCTION}

Levobupivacaine is an isolated enantiomer of the long-acting local anaesthetic bupivacaine $(1,2)$. Levobupivacaine is less arrhythmogenic than the same doses of bupivacaine up to 75-122 mg. Additionally levobupivacaine affects corrected QT interval and QRS duration lesser than that bupivacaine does. Levobupivacaine has become a preferred agent in regional anaesthesia because of better safety profile (3-8). Minimum intrathecal dose of levobupivacaine with maximal anaesthetic efficacy without prolonged hospital stay was reported as $12 \mathrm{mg}$ (5).

Combination of local anaesthetics and opioids are commonly used in order to achieve more effective sensory and motor block. Fentanyl and morphine are most commonly used opioids in subarachnoid block (9-12). Various studies reported sufficient postoperative analgesia with intrathecal morphine between 100-200 $\mu \mathrm{g}$ doses (13-15).

In this study we compared the quality of anaesthesia and analgesia in addition to surgeon and patient satisfactions with different doses of morphine combined with levobupivacaine in patients undergoing elective inguinal hernia repair.

\section{MATERIAL and METHODS}

After obtaining ethical approval from Gazi University Ethics Committee and written informed consent from the patients; sixty patients aged between 1875 undergoing elective unilateral inguinal hernia repair were enrolled for the study. Physical status of all patients were either American Society of Anesthesiologists (ASA) I or II. Demographic data were recorded. Patients who refused regional anaesthesia, with motor and/or sensory deficit or any neurological sequel, advanced heart disease (advanced aortic or mitral valve stenosis, cardiomyopathy etc.), bleeding disorder, recent history of nonsteroid anti-inflammatory drug, Acetylsalicylic Acid (ASA) usage or low molecular weight heparin usage in 12 hours or heparin usage in 4 hours period before intervention, skin infection at injection area, sepsis, coma, psycho-motor disorders, any sensitivity to study drugs or refused to be enrolled into study were excluded from study.

Patients were randomly divided into three groups: $12 \mathrm{mg} /$ total volume 2.5 $\mathrm{ml}$ levobupivacaine only (Group L), $12 \mathrm{mg}$ levobupivacaine $(0.5 \%, 2.4 \mathrm{ml}$ Chirocaine $^{*}$ Nycomed Pharma/Norvey $+0.1 \mathrm{ml}$ distilled water, total volume $2.5 \mathrm{ml}$ ) - with $50 \mu \mathrm{g}$ morphine (Group $\left.\mathrm{LM}_{50}\right), 12 \mathrm{mg} /$ total volume $2.5 \mathrm{ml}$ levobupivacaine with $100 \mu \mathrm{g}$ morphine group (Group LM $_{100}$ ). Intravenous bolus infusion of $10 \mathrm{ml} / \mathrm{kg}$ lactated Ringer's solution was administered in 15 minutes before anaesthesia induction. All patients had non-invasive blood pressure (mean, systolic and diastolic blood pressures) monitoring, pulse oximetry measurement of blood oxygen saturation $\left(\mathrm{SpO}_{2}\right)$ and electrocardiography. Spinal anaesthesia was performed at the L3 - L4 levels with a 27-gauge Pencil point spinal needle (Exelint, spinal needles, $25 G \times 3 \frac{1}{2}$, USA) when the patient was in sitting position. After a free flow of cerebrospinal fluid was observed, a total volume of $2.5 \mathrm{ml}$ of spinal solution was administered to each patient over approximately $30 \mathrm{~s}$. Patients were moved to the supine position immediately after recording the number of successful attempts. Sensory block level was assessed using pin prick test and motor block level was determined by using modified Bromage scale.
Vital signs (heart rate, $\mathrm{SpO}_{2}, \mathrm{SAP}, \mathrm{DAP}, \mathrm{MAP}$ ) sensorial and motor block levels, side effects and drug therapies were recorded at 0., 2., 4., 6., 8., 10., 15., 20., 25., 30., 35., 40., 45., 50., 55., 60., 75., 90., 105., 120th minutes and end of the operation. Achieving sensorial block at $T_{8}$ was considered as sufficient block level.

Patient and surgeon satisfaction levels were assessed using a 5 point scale (0-not good, 1-nearly good, 2-good, 3-very good, 4-perfect) at the end of surgery. Postoperative $\mathrm{HR}, \mathrm{SAP}, \mathrm{DAP}, \mathrm{MAP}, \mathrm{SpO}_{2}$, side effects, treatments, sensory and motor block levels were recorded for 60 minutes with 10 minutes intervals postoperatively. At the end of 60 minutes period, patients with normal vital signs and sensory block highest at $T_{8}$ were questioned about when the patient needed analgesic drug. Time of sensory and motor block, first mobilization, urination and defacation were recorded.

Episodes of bradycardia (heart rate $<50$ beats/min) and peri-operative hypotension (SAP $<20 \%$ of baseline) were recorded and treated with boluses of fluid $50(\mathrm{ml} / \mathrm{min})$ however cases resistant to fluid administration were treated with intravenous ephedrine $(5 \mathrm{mg})$.

Patients with postoperative nausea and vomiting were treated with metoclopramide $10 \mathrm{mg}$ iv while tenoxicam $20 \mathrm{mg}$ iv was administered to patients with a Verbal Numeric Rating Scale (VNS) score 4 at postoperative period.

Patients were closely followed until hospital discharge for headache, back pain, urinary retention, motor deficit or leg pain, urinary or fecal incontinence, nausea, vomiting, hypotension and bradycardia.

\section{Statistical Analysis}

Statistical analysis was performed using SPSS version 12.0 (SPSS Inc. Chicago, IL, USA). Measurable variables were analysed using KolmogorovSmirnov test while One way ANOVA was used in order to analyse between group differences for age, weight, height, duration of operation, number of lumbar punctures, time to attaining sufficient anaesthesia duration for surgery, amount of administered fluid at perioperative period, time to achieving maximum sensory and motor block, time to two segment regression, duration of analgesia, time to first ambulation, urination, discharge from hospital, satisfaction levels of patients and surgeons. Between group differences were compared using Posthoc Bonferroni test.

Gender, ASA status, complications occurred during perioperative and postoperative periods, number of patients treated with ephedrine or atropine were analysed using Chi-square or Fisher's exact tests. Levels of sensory and motor blocks in addition to maximum block levels were analysed using Kruskal-Wallis test. Mann-Witney $U$ test was used. Mann Witney U test was used in order to analyse significant differences determined in Kruskal Wallis test. A $P$-value $<0.05$ was considered statistically significant.

\section{RESULTS}

There was no significant difference between groups for demographic variables. Similarly, duration of operation, number of interventions, time to achieving sufficient sensory block, totally volume of administered intravenous fluids were indifferent between study groups (Table 1).

Table 1. Duration of surgery, number ofattempts, time to achieving sufficient anaesthesia level, total volume of pre-operative administered fluids (Mean \pm SD (MinMax)

\begin{tabular}{|c|c|c|c|}
\hline & $\begin{array}{l}\text { Group L } \\
(n=20)\end{array}$ & $\begin{array}{l}\text { Group LM } \mathbf{L M}_{50} \\
(\mathrm{n}=20)\end{array}$ & $\begin{array}{l}\text { Group LM } \text { LM }_{100} \\
\quad(n=20)\end{array}$ \\
\hline Duration of surgery(min) & 72. $85 \pm 22.70(65-115)$ & $79.20 \pm 12.68(65-100)$ & $86.35 \pm 11.91(65-114)$ \\
\hline Number of punctions & $1,20 \pm 0,41(1-2)$ & $1,30 \pm 0,47(1-2)$ & $1,10 \pm 0,31(1-2)$ \\
\hline $\begin{array}{l}\text { Time to achieving sufficient }\left(T_{8}\right) \\
\text { anaesthesia level (min) }\end{array}$ & $12,70 \pm 4,92(6-20)$ & $12,40 \pm 3,80(6-20)$ & $12,15 \pm 3,13(8-20)$ \\
\hline $\begin{array}{l}\text { Total volume of preoperatively } \\
\text { administered fluids }(\mathrm{mL})\end{array}$ & $587,50 \pm 162,12(300-1000)$ & $515,00 \pm 67,08(400-700)$ & $587,50 \pm 113,41(450-900)$ \\
\hline Total volume of administered fluids ( $\mathrm{mL}$ ) & $1360,00 \pm 177,66(1000-1650)$ & $1417,00 \pm 224,06(1000-2000)$ & $1505,00 \pm 158,03(1200-1800)$ \\
\hline
\end{tabular}

There was no significant difference between three groups for preoperative and postoperative mean HR. MAP for Group L, Group LM $\mathrm{LM}_{50}$ and Group $\mathrm{LM}_{100}$ were found significantly lower than that in control values at different time points $(p<0.05)$ (Table 2$)$. 
Table 2. Distribution of group means of mean arterial blood pressure (mmHg) [Mean \pm SD (Min-Max)]

\begin{tabular}{|c|c|c|c|}
\hline Time & $\begin{array}{c}\text { Group L } \\
(n=20)\end{array}$ & $\begin{array}{c}\text { Group LM } \text { LM }_{50} \\
(n=20)\end{array}$ & $\begin{array}{c}\text { Group LM } \text { LM }_{100} \\
(n=20)\end{array}$ \\
\hline Control & $103,70 \pm 15,09(77-137)$ & $107,60 \pm 17,11(69-133)$ & $101,90 \pm 11,11(84-128)$ \\
\hline $0 . \min$ & $111,10 \pm 14,12(90-135)$ & $103,80 \pm 19,03(64-133)$ & $103,95 \pm 16,51(73-136)$ \\
\hline 2. $\min$ & $104,45 \pm 14,25(88-136)$ & $97,95 \pm 15,86(71-132)$ & $103,10 \pm 12,30(84-124)$ \\
\hline 4. $\min$ & $104,90 \pm 16,77(77-135)$ & $98,05 \pm 14,42(72-130)$ & $96,70 \pm 17,00(49-126)$ \\
\hline 6. $\min$ & $100,40 \pm 13,23(78-122)$ & $99,00 \pm 16,08(65-136)$ & $98,85 \pm 16,08(60-130)$ \\
\hline 8. $\min$ & $97,55 \pm 15,61(75-123)$ & $94,20 \pm 13,22(70-125)$ & $96,05 \pm 15,31(62-128)$ \\
\hline 10. $\min$ & $94,40 \pm 13,34(74-123)$ & $94,55 \pm 13,78(65-124)$ & $93,55 \pm 12,18(73-122)$ \\
\hline 15. $\min$ & $91,00 \pm 15,97+(60-125)$ & $91,00 \pm 14,41(70-131)$ & $93,90 \pm 12,61(70-123)$ \\
\hline 20. $\min$ & $90,15 \pm 18,24+(61-131)$ & $88,70 \pm 12,83+(75-122)$ & $91,75 \pm 12,99(73-121)$ \\
\hline 25. $\min$ & $90,65 \pm 16,45(64-131)$ & $87,90 \pm 13,41+(69-113)$ & $89,95 \pm 15,18(74-131)$ \\
\hline 30. $\mathrm{min}$ & $92,90 \pm 17,02(63-126)$ & $85,85 \pm 11,19+(70-105)$ & $87,55 \pm 12,99+(62-111)$ \\
\hline 35. $\min$ & $91,70 \pm 16,14(66-125)$ & $89,35 \pm 11,65+(71-112)$ & $85,80 \pm 10,97+(66-107)$ \\
\hline 40. $\min$ & $94,40 \pm 16,22(63-124)$ & $87,65 \pm 10,84+(71-113)$ & $89,80 \pm 10,97+(66-107)$ \\
\hline 45. $\min$ & $93,35 \pm 16,73(57-119)$ & $87,50 \pm 11,24+(74-109)$ & $88,95 \pm 12,83+(65-112)$ \\
\hline 50. $\mathrm{min}$ & $95,89 \pm 14,86(62-117)$ & $89,90 \pm 12,13+(72-113)$ & $87,15 \pm 12,15+(68-111)$ \\
\hline 55. $\min$ & $94,79 \pm 17,92(64-131)$ & $90,35 \pm 12,25(72-120)$ & $88,40 \pm 10,77+(68-106)$ \\
\hline 60. $\min$ & $92,78 \pm 15,86(66-127)$ & $91,60 \pm 15,68(70-127)$ & $87,45 \pm 14,17+(61-111)$ \\
\hline 75. $\min$ & $94,06 \pm 16,35(59-123)$ & $88,50 \pm 11,65+(75-114)$ & $90,60 \pm 14,36(70-121)$ \\
\hline $\begin{array}{l}\text { At the end of } \\
\text { operation }\end{array}$ & $97,10 \pm 15,19(66-131)$ & $92,75 \pm 14,22(76-137)$ & $89,40 \pm 10,59(74-124)$ \\
\hline
\end{tabular}

Mean sensory block level of patients in Group $L$ at 20th minute was significantly lower than those in Group $\mathrm{LM}_{50}$ and Group $\mathrm{LM}_{100}(\mathrm{p}<0.05)$, (Table 3). Also mean sensory block level of Group $\mathrm{LM}_{100}$ between 30-60 minutes was significantly higher than those in Group L and Group LM50 $(\mathrm{p}<0.05)$, (Table 3).

Table 3: Median values of dermatomal sensory block levels [Median(Min-Max)]

\begin{tabular}{|c|c|c|c|}
\hline Time & $\begin{array}{c}\text { Group L } \\
(n=20)\end{array}$ & $\begin{array}{c}\text { Group LM } \\
\quad(n=20)\end{array}$ & $\begin{array}{c}\text { Group } \mathrm{LM}_{100} \\
(\mathrm{n}=20)\end{array}$ \\
\hline 2. $\min$ & $\mathrm{L}_{2}\left(\mathrm{~L}_{4}-\mathrm{T}_{12}\right)$ & $\mathrm{L}_{2}\left(\mathrm{~L}_{4}-\mathrm{T}_{10}\right)$ & $L_{2}\left(L_{3}-L_{1}\right)$ \\
\hline 4. $\min$ & $\mathrm{L}_{1}+\left(\mathrm{L}_{3}-\mathrm{T}_{10}\right)$ & $\mathrm{L}_{1}+\left(\mathrm{L}_{3}-\mathrm{T}_{9}\right)$ & $\mathrm{L}_{1}+\left(\mathrm{L}_{2}-\mathrm{T}_{12}\right)$ \\
\hline 6. $\min$ & $\mathrm{T}_{12}+\left(\mathrm{L}_{2}-\mathrm{T}_{8}\right)$ & $\mathrm{T}_{12}+\left(\mathrm{L}_{2}-\mathrm{T}_{8}\right)$ & $\mathrm{T}_{12}+\left(\mathrm{L}_{1}-\mathrm{T}_{10}\right)$ \\
\hline 8. $\min$ & $\mathrm{T}_{10}+\left(\mathrm{L}_{1}-\mathrm{T}_{6}\right)$ & $\mathrm{T}_{10}+\left(\mathrm{L}_{1}-\mathrm{T}_{6}\right)$ & $T_{10}+\left(T_{12}-T_{8}\right)$ \\
\hline 10. $\min$ & $T_{9}+\left(T_{12}-T_{6}\right)$ & $T_{9}+\left(T_{12}-T_{6}\right)$ & $T_{8}+\left(T_{10}-T_{6}\right)$ \\
\hline 15. $\min$ & $T_{8}+\left(T_{10}-T_{6}\right)$ & $T_{8}+\left(T_{10}-T_{5}\right)$ & $T_{8}+\left(T_{8}-T_{6}\right)$ \\
\hline 20. $\min$ & $\mathrm{T}_{8}+\left(\mathrm{T}_{8}-\mathrm{T}_{6}\right)$ & $T_{6} *,+\left(T_{8}-T_{5}\right)$ & $T_{6} *,+\left(T_{8}-T_{5}\right)$ \\
\hline 25. $\min$ & $T_{6}+\left(T_{8}-T_{5}\right)$ & $T_{6}+\left(T_{8}-T_{5}\right)$ & $T_{6}+\left(T_{7}-T_{4}\right)$ \\
\hline 30. $\min$ & $T_{6+}\left(T_{8}-T_{5}\right)$ & $T_{6}+\left(T_{8}-T_{5}\right)$ & $T_{5} *, \S\left(T_{6}-T_{4}\right)$ \\
\hline 35. $\min$ & $\mathrm{T}_{6}+\left(\mathrm{T}_{8}-\mathrm{T}_{4}\right)$ & $T_{6}+\left(T_{8}-T_{5}\right)$ & $T_{5} *, \S,+\left(T_{6}-T_{4}\right)$ \\
\hline 40. $\min$ & $\mathrm{T}_{6}+\left(\mathrm{T}_{8}-\mathrm{T}_{4}\right)$ & $T_{6}\left(T_{8}-T_{5}\right)$ & $T_{5} *, \S\left(T_{6}-T_{4}\right)$ \\
\hline 45. $\min$ & $T_{6}+\left(T_{10}-T_{4}\right)$ & $T_{6}\left(T_{7}-T_{5}\right)$ & $T_{5} *, \S\left(T_{6}-T_{4}\right)$ \\
\hline 50. $\min$ & $T_{6}+\left(T_{10}-T_{4}\right)$ & $\mathrm{T}_{6}+\left(\mathrm{T}_{7}-\mathrm{T}_{5}\right)$ & $T_{5} *, \S,+\left(T_{6}-T_{4}\right)$ \\
\hline 55. $\min$ & $T_{6}+\left(T_{11}-T_{4}\right)$ & $T_{6}+\left(T_{7}-T_{5}\right)$ & $T_{5} *, \S,+\left(T_{6}-T_{4}\right)$ \\
\hline 60. $\min$ & $\mathrm{T}_{6}+\left(\mathrm{T}_{8}-\mathrm{T}_{4}\right)$ & $T_{6}+\left(T_{7}-T_{5}\right)$ & $T_{5} *, \S,+\left(T_{6}-T_{4}\right)$ \\
\hline 75. $\min$ & $\mathrm{T}_{6}+\left(\mathrm{T}_{8}-\mathrm{T}_{4}\right)$ & $T_{6}+\left(T_{7}-T_{5}\right)$ & $T_{6}+\left(T_{6}-T_{5}\right)$ \\
\hline $\begin{array}{l}\text { At the end of } \\
\text { surgery }\end{array}$ & $T_{7}+\left(T_{11}-T_{6}\right)$ & $T_{7}+\left(T_{8}-T_{6}\right)$ & $T_{7}+\left(T_{8}-T_{5}\right)$ \\
\hline
\end{tabular}

* : $\mathrm{p}<0,05$ (Compared to Group L)

$\S:$ : $<0,05$ (Compared to Group LM $_{50}$ )

${ }^{+}: \mathrm{P}<0,05$ (Compared to withingroup levels at 2 th minute)

Incidence and percentages of side effects that observed in three groups were presented in Table 4. Bradycardia was seen in three patients and hypotension in two patients in Group $\mathrm{LM}_{50}$ while 2 patients suffered from bradycardia and 1 patient from hypotension in Group $\mathrm{LM}_{100}$. In Group L, only one patient suffered from hypotension (Table 4). 
Table 4: Perioperative side-effects [n (\%)]

\begin{tabular}{lccc}
\hline & $\begin{array}{c}\text { Group L } \\
(\mathrm{n}=20)\end{array}$ & $\begin{array}{c}\text { Group LM } \\
(\mathrm{n}=20)\end{array}$ & $\begin{array}{c}\text { Group LM } \\
(\mathrm{n}=20)\end{array}$ \\
Hypotension & $1(5)$ & $2(10)$ & $1(5)$ \\
Bradycardia & $0(0)$ & $3(15)$ & $2(10)$ \\
Nausea & $0(0)$ & $0(0)$ & $0(0)$ \\
Vomiting & $0(0)$ & $0(0)$ & $0(0)$ \\
Respiratory depression & $0(0)$ & $0(0)$ & $0(0)$ \\
\hline
\end{tabular}

Mean arterial pressure levels at different time points during postoperative period were similar for all study groups. In addition, MAP levels of three groups were found similar with that in control values (Table 5).

Table 5. Postoperative mean arterial blood pressure values ( $\mathrm{mmHg}$ ) [Mean $\pm \mathrm{SD}$ (Min-Max)]

\begin{tabular}{lccc}
\hline Time & \multicolumn{1}{c}{$\begin{array}{c}\text { Group } L \\
(\mathrm{n}=20)\end{array}$} & $\begin{array}{c}\text { Group LM } \\
(\mathrm{n}=20)\end{array}$ & $\begin{array}{c}\text { Group LM } \\
(\mathrm{n}=20)\end{array}$ \\
\hline Postoperative 0. $\min$ (Control) & $101,10 \pm 12,73(79-125)$ & $94,15 \pm 15,14(72-121)$ & $95,65 \pm 14,35(69-121)$ \\
10. $\min$ & $99,85 \pm 13.52(81-134)$ & $93,15 \pm 17,39(52-126)$ & $94,70 \pm 13,86(69-121)$ \\
20. $\min$ & $95,45 \pm 12,50(67-116)$ & $97,00 \pm 15,50(73-125)$ & $95,10 \pm 12,35(70-118)$ \\
30. $\min$ & $97,00 \pm 13,60(81-136)$ & $93,55 \pm 12,77(75-117)$ & $94,05 \pm 14,02(64-131)$ \\
40. $\min$ & $92,85 \pm 11,03(77-115)$ & $95,90 \pm 14,23(72-121)$ & $91,95 \pm 13,22(72-124)$ \\
50. $\min$ & $91,70 \pm 10,04(81-117)$ & $92,05 \pm 10,28(80-107)$ & $92,10 \pm 11,55(75-117)$ \\
60. $\min$ & $94,70 \pm 13,22(71-121)$ & $94,30 \pm 11,60(77-119)$ & $93,90 \pm 11,79(73-119)$ \\
\hline
\end{tabular}

Median values of sensory block levels at postoperative 20th and 30th minutes in Group $\mathrm{LM}_{50}$ and Group $\mathrm{LM}_{100}$ were significantly higher than in Group L ( $p<0.05$ ), (Table 6). Postoperative sensory block levels at 20th, 30th, 40th, 50th and 60th minutes in Group L were significantly lower than that at
Oth minute postoperatively $(\mathrm{p}<0.05)$. In Group $\mathrm{LM}_{50}$ and Group $\mathrm{LM}_{100}$ sensory block levels at 30th, 40th, 50th and 60th minutes were significantly lower than that at 0 th minutes postoperatively $(p<0.05)$, (Table 6).

Table 6. Postoperative dermatomal sensory block levels [Median (Min-Max)]

\begin{tabular}{|c|c|c|c|}
\hline Time & $\begin{array}{l}\text { Group L } \\
(n=20)\end{array}$ & $\begin{array}{l}\text { Group LMs0 } \\
(n=20)\end{array}$ & $\begin{array}{l}\text { Group LM }{ }_{100} \\
\quad(n=20)\end{array}$ \\
\hline Postoperative 0. $\min$ (Control) & $T_{8}\left(T_{11}-T_{6}\right)$ & $T_{8}\left(T_{8}-T_{6}\right)$ & $T_{8}\left(T_{8}-T_{6}\right)$ \\
\hline 10. $\min$ & $T_{8}\left(T_{12}-T_{6}\right)$ & $T_{8}\left(T_{10}-T_{6}\right)$ & $T_{8}\left(T_{10}-T_{7}\right)$ \\
\hline 20. $\min$ & $\mathrm{T}_{10}+\left(\mathrm{T}_{12}-\mathrm{T}_{6}\right)$ & $\mathrm{T}_{8} *\left(\mathrm{~T}_{10}-\mathrm{T}_{7}\right)$ & $\mathrm{T}_{8} *\left(\mathrm{~T}_{10}-\mathrm{T}_{7}\right)$ \\
\hline 30. $\min$ & $\mathrm{T}_{10}+\left(\mathrm{T}_{12}-\mathrm{T}_{6}\right)$ & $\mathrm{T}_{9} *,+\left(\mathrm{T}_{10}-\mathrm{T}_{7}\right)$ & $T_{9} *,+\left(T_{10}-T_{8}\right)$ \\
\hline 40. $\min$ & $\mathrm{T}_{10}+\left(\mathrm{T}_{12}-\mathrm{T}_{6}\right)$ & $\mathrm{T}_{10}+\left(\mathrm{T}_{10}-\mathrm{T}_{8}\right)$ & $\mathrm{T}_{10}+\left(\mathrm{T}_{10}-\mathrm{T}_{8}\right)$ \\
\hline 50. $\min$ & $\mathrm{T}_{10}+\left(\mathrm{T}_{12}-\mathrm{T}_{8}\right)$ & $\mathrm{T}_{10}+\left(\mathrm{T}_{10}-\mathrm{T}_{8}\right)$ & $\mathrm{T}_{10}+\left(\mathrm{T}_{10}-\mathrm{T}_{9}\right)$ \\
\hline 60. $\min$ & $\mathrm{T}_{10}+\left(\mathrm{T}_{12}-\mathrm{T}_{8}\right)$ & $\mathrm{T}_{10}+\left(\mathrm{T}_{10}-\mathrm{T}_{8}\right)$ & $T_{10}+\left(T_{10}-T_{9}\right)$ \\
\hline
\end{tabular}

* : $p<0.05$ (Compared to Group L)

${ }^{+}: p<0.05$ (Withingroup comparison)

The mean time required to achieve maximum sensory block was similar for all study groups. However, the mean time required to achieve maximum motor block were significantly shorter in Group $\mathrm{LM}_{50}$ and Group $\mathrm{LM}_{100}$ than that in Group $L(p=0.008$ and $p=0.001$ respectively). The duration of motor block was significantly longer in Group $\mathrm{LM}_{50}$ and Group $\mathrm{LM}_{100}$ than that in Group $L(p=0.020$ and $p=0.019$ respectively), (Table 7$)$.

Table 7. Time to onset and duration of sensory and motor blocks [Mean \pm SD (Min-Max)

\begin{tabular}{lccc}
\hline & $\begin{array}{c}\text { Group L } \\
(\mathrm{n}=20)\end{array}$ & $\begin{array}{c}\text { Group LM } \\
(\mathrm{n}=20)\end{array}$ & $\begin{array}{c}\text { Group LM } \\
(\mathrm{n}=20)\end{array}$ \\
\hline Time to maximum sensory block $\mathrm{min})$ & $22,55 \pm 10,34(8-45)$ & $22,65 \pm 8,04(8-40)$ \\
Time to maximum motor block $(\mathrm{min})$ & $11,80 \pm 5,62(6-25)$ & $8,50 \pm 1,43 *(6-10)$ \\
Duration of sensory block $(\min )$ & $305,45 \pm 86,64(165-580)$ & $347,25 \pm 54,66(270-450)$ & $7,80 \pm 1,44 *(6-10)$ \\
Duration of motor block $(\min )$ & $268,50 \pm 68,80(162-475)$ & $316,25 \pm 54,69 *(240-420)$ & $340,65 \pm 30,53(300-408)$ \\
\hline
\end{tabular}

* : $\mathrm{p}<0.05$ (Compared to Group L)

The mean time for first postoperative analgesic demand in Group $L$ was significantly shorter than those in Group LM $\mathrm{L}_{50}$ and Group $\mathrm{LM}_{100}(\mathrm{p}=0.001$ and $p<0.0001$ respectively). Mean time for first analgesic demand in Group $L_{50}$ was also significantly shorter than that in Group $\mathrm{LM}_{100}(\mathrm{p}=0.001)$, (Table 8). Time to first mobilization, urination were significantly shorter in Group $L$ than those in other two study groups ( $p<0.05)$, (Table 8). Also time for first passing gas was significantly shorter in Group $\mathrm{LM}_{50}$ than that in Group $\mathrm{LM}_{100}$ $(p<0.0001)$, (Table 8). However duration of hospital stay for all study groups were found indifferent to each other (Table 8). 
Table 8. Parameters at early postoperative period related with segmental anaesthesia regression and other signs of recovery [Mean \pm SD (Min-Max)

\begin{tabular}{|c|c|c|c|}
\hline & $\begin{array}{l}\text { Group L } \\
(n=20)\end{array}$ & $\begin{array}{l}\text { Group LM } \mathbf{L}_{50} \\
\quad(n=20)\end{array}$ & $\begin{array}{l}\text { Group } L^{2} M_{100} \\
\quad(n=20)\end{array}$ \\
\hline $\begin{array}{l}\text { Mean time for two segment } \\
\text { anaesthesia regression ( } \mathrm{min}) \\
\text { Mean time for first }\end{array}$ & $85,55 \pm 20,36(45-120)$ & $97,75 \pm 10,32 *(80-120)$ & $98,25 \pm 9,90 *(90-120)$ \\
\hline $\begin{array}{l}\text { postoperative analgesic } \\
\text { demand ( } \mathrm{min})\end{array}$ & $362,70 \pm 94,73(220-640)$ & $486,75 \pm 104,83 *(320-630)$ & $620,75 \pm 115,91 * * *(355-810)$ \\
\hline $\begin{array}{l}\text { Mean time for first mobilisation } \\
\text { (min) }\end{array}$ & $541,05 \pm 59,14(447-660)$ & $614,25 \pm 65,16 *(520-720)$ & $636,90 \pm 86,14 *(480-775)$ \\
\hline $\begin{array}{l}\text { Mean time for first urination } \\
\text { (min) }\end{array}$ & $557,25 \pm 63,03(450-675)$ & $637,35 \pm 85,61 *(530-845)$ & $684,00 \pm 86,57 *(540-800)$ \\
\hline $\begin{array}{l}\text { Mean time for first gas passing } \\
\text { (min) }\end{array}$ & $648,75 \pm 97,95(450-820)$ & $893,25 \pm 126,58 *(600-1140)$ & $1173,75 \pm 105,92^{*}, * *(1000-1400)$ \\
\hline $\begin{array}{l}\text { Mean time for hospital } \\
\text { discharge (min) }\end{array}$ & $648,75 \pm 97,95(450-820)$ & $893,25 \pm 126,58(600-1140)$ & $1173,75 \pm 105,92(1000-1400)$ \\
\hline
\end{tabular}

$*: p<0,05$ (compared to Group L),

$* *: p<0,05$ (compared to Group LMso)

Patient satisfaction levels were lower in Group $L$ than those in other groups ( $p=0.0001, p<0.0001$ respectively). In contrast highest patient satisfaction levels were recorded in Group $\mathrm{LM}_{100}$ when compared with Group $\mathrm{LM}_{50}$ $(p=0.004)$, (Table 9). Similar results for surgeon satisfaction levels were identified for all groups (Table 9). There was no significant difference between groups in terms of postoperative complications (Table 9). Although statistically insignificant, urinary retention incidence was higher in levobupivacaine plus morphine groups than that observed in levobupivacaine group ( $p>0.05)$

Table 9. Patient and surgeon satisfaction levels and postoperative side effects [Mean \pm SD (Min-Max), n (\%)]

\begin{tabular}{lccc}
\hline & $\begin{array}{c}\text { Group L } \\
(\mathrm{n}=20)\end{array}$ & $\begin{array}{c}\text { Group LM } \\
(\mathrm{n}=20)\end{array}$ & $\begin{array}{c}\text { Group LM } \\
\text { (n) } \\
(\mathrm{n}=20)\end{array}$ \\
\hline Patient satisfaction & $2,20 \pm 0,41(2-3)$ & $2,80 \pm 0,41^{*}(2-3)$ & $3,05 \pm 0,22^{*}, * *(3-4)$ \\
Surgeon satisfaction & $1,90 \pm 0,31(1-2)$ & $2,25 \pm 0,44^{*}(2-3)$ & $3,05 \pm 0,22^{*}, * *(3-4)$ \\
Hypotension & $0(0)$ & $0(0)$ & $0(0)$ \\
Bradycardia & $0(0)$ & $0(0)$ & $0(0)$ \\
Nausea & $0(0)$ & $0(0)$ & $0(0)$ \\
Vomiting & $0(0)$ & $0(0)$ & $0(0)$ \\
Respiratory depression & $0(0)$ & $(0)$ & $(0)$ \\
Urinary retention & $0(0)$ & $3(15)$ & $5(25)$ \\
\hline
\end{tabular}

* : $p<0.05$ (Compared to Group L)

$* *$ : $p<0.05$ (Compared to Group LM LM)

\section{DISCUSSION}

Analgesic and anaesthetic effects of levobupivacaine start in 8-11 minutes after intrathecal administration, motor block -at a ratio of $83-100 \%$ - is achieved with a sensory block duration of 360-390 minutes (1-3). Various levobupivacaine doses are used in different surgical procedures. In orthopaedic surgery recommended dose of isobaric levobupivacaine is between 11.7-17.5 $\mathrm{mg}(6,7,16)$. While in unilateral spinal block $5 \mathrm{mg}$ dose is recommended (17). In urological surgery recommended dose is between $12.5-13 \mathrm{mg}$ with isobaric solution $(8,18)$. While in mixed lower abdominal or orthopaedic surgeries $15 \mathrm{mg}$ isobaric or hyperbaric levobupivacaine solutions are being used (19).

We found lower block levels and shorter anaesthesia duration with $12 \mathrm{mg}$ levobupivacaine only group when compared with previous studies. Alley et al (5) used $12 \mathrm{mg}$ hyperbaric levobupivacaine solution and they found higher sensory block (T5 vs T6), lesser time for achieving maximum sensory block (15.0 \pm 9.0 vs $22.5 \pm 10.0$ minutes) and time need for two-segment regression of sensory block $(62.0 \pm 30.0$ vs $85.5 \pm 20.0 \mathrm{~min}$ ). The differences between two studies may be explained with different features (hyperbaric vs isobaric) of solutions used in studies.

Casati et al (20) compared hyperbaric levobupivacaine ( $8 \mathrm{mg}$ ), bupivacaine and ropivacaine in unilateral inguinal hernia repair operations and they found similar maximum sensory block levels $\left[T_{6}\left(T_{5-12}\right)\right]$ with three agent during unilateral spinal block. Additionally they reported similar duration of spinal anaesthesia with levobupivacaine and bupivacaine while shorter duration with ropivacaine. We found similar maximum sensory block level with $12 \mathrm{mg}$ isobaric levobupivacaine $\left(T_{6} ; T_{4-8}\right)$ while longer anaesthesia duration.
The difference may be arisen due to different doses and barite of used solutions in addition to unilateral block used in the study conducted by Casati et al. in their study (midazolam $(0.03 \mathrm{mg} / \mathrm{kg}$ ) iv.) preoperatively and sedation with iv propofol was needed in $30 \%$ of patients (20). This data indicates that $8 \mathrm{mg}$ hyperbaric doses of levobupivacaine or bupivacaine only may not provide sufficient anaesthesia level in inguinal hernia operations. We used $12 \mathrm{mg}$ levobupivacaine in this study however surgeon and patient satisfaction levels were significantly lower in levobupivacaine only group compared with those determined in other groups. Also surgeon and patient satisfaction levels in $\mathrm{LM}_{100}$ Group were significantly higher than those in other groups. These results suggest that $12 \mathrm{mg}$ isobaric levobupivacaine is the minimal intratechal dose that provide sufficient analgesia in unilateral inguinal hernia repair while optimal levobupivacaine only dose has to be higher than $12 \mathrm{mg}$ because of determined higher satisfaction levels in adjuvant morphine groups in our study.

Demiraran et al. (21) showed unchanged block levels with intrathecal morphine administration. In contrast to this study we showed significant higher block levels with morphine plus levobupivacaine group. In the study conducted by Demiraran et al. (21), $5 \mathrm{mg}$ hyperbaric bupivacaine plus $160 \mu \mathrm{g}$ morphine (a total volume of $1.25 \mathrm{ml}$ ) were used for unilateral spinal block in orthopaedic surgery. We suggest that unilateral spinal block and lower solution volume (1.25 vs $2.5 \mathrm{ml}$ ) and dose of local anaesthetic $(5 \mathrm{mg})$ used in the study might lead to lower block level. In these circumstances possible morphine effects on block level might be inhibited.

Gupta et al. (10) reported sufficient anaesthesia level with bupivacaine 7.5 mg plus fentanyl $25 \mu \mathrm{g}$ in inguinal hernia repair although they used sedative agents -fentanyl and propofol ( $5 \%$ and $45 \%$ respectively)- in patients $7.5 \mathrm{mg}$ bupivacaine group. This data indicates insufficient block level with suboptimal local anaesthetic doses despite adjuvant opioid administration. 
Girgin et al. (22) compared 5 vs $7.5 \mathrm{mg}$ levobupivacaine plus $25 \mu \mathrm{g}$ fentany in inguinal hernia repair and concluded that similar sensory and motor block levels with two different doses while regression time of sensory block was significantly shorter in low dose group. In this study significantly lower doses were used than that were in our study but they used midazolam at a dose of $0.03 \mathrm{mg} / \mathrm{kg}$ iv preoperatively.

Time to first analgesic requirement was significantly longer in Group $\mathrm{LM}_{50}$ and $\mathrm{LM}_{100}$ than that in Group L. Also in Group $\mathrm{LM}_{100}$ the duration was longer than that in Group $\mathrm{LM}_{50}$. We found that quality of analgesia with morphine was significantly higher and dose dependent. Another important finding of our study is insignificantly affected postoperative mobilization time after adjuvant morphine administration. Even as in Group $\mathrm{LM}_{100}$ higher dose morphine was related with motor block without prolonged mobilization time. Similarly Kusunemi et al. (23) showed prolonged time for regression of sensory block with $25 \mu \mathrm{g}$ fentanyl added to $10 \mathrm{mg}$ bupivacaine compared with $25 \mu \mathrm{g}$ fentanyl plus $5,7.5 \mathrm{mg}$ bupivacaine in urological surgery without any prolongation in time to hospital discharge. In another study Demiraran et al. (21) showed sufficient postoperative analgesia level with $160 \mu \mathrm{g}$ morphine added to local anaesthetic without elongated mobilization time in orthopaedic surgery.

Respiratory depression is a well-known side effect after intrathecal administration of morphine at 200-300 $\mu$ g doses (24). In a study conducted by Slappendel et al. (25) desaturation of peripheral oxygen levels $(90 \%)$ without any acidosis in $10-20 \%$ of patients undergoing orthopaedic surgery were reported after intrathecal bupivacaine $20 \mathrm{mg}$ plus $25-200 \mu \mathrm{g}$ morphine. In contrast neither respiratory depression nor any neurological complications were seen in both of morphine groups in our study. Similarly various studies showed sufficient safety profile without any respiratory depression with morphine at different doses between 50-300 $\mu \mathrm{g}(9,15,26)$.

Nausea-vomiting, itching and urinary retention are common side effects of intrathecal morphine $(24,27)$. There are various studies that indicate insignificant increase in postoperative nausea-vomiting after high doses of morphine such as $300 \mu \mathrm{g}$ intrathecally $(9,15,25,26)$. In a similar manner it was shown that postoperative itching following intrathecal morphine usage is dose dependent and morphine doses above $200 \mu \mathrm{g}$ are strongly correlated with increased itching rates $(9,25)$. In our study, we couldn't report postoperative nausea-vomiting and itching. This might be a consequence of relatively lower morphine doses used in our study.

Hemodynamic disturbances especially hypotension and bradycardia are often seen following intrathecal morphine administration $(24,28)$. However only mild hypotension (intragroup, intraoperative systolic, diastolic and mean arterial pressure) -correlated with relatively higher block levels- was reported in several patients during study. As a consequence we can conclude that intrathecal morphine -as an adjuvant- at doses we used has minimal and tolerable hemodynamic side effect profile in inguinal hernia repair.

Urinary retention is a major limiting factor for spinal block in outpatient surgery due to elongated time for urination and hospital discharge (29-32). Because of higher block level requirements in lower abdominal surgeries such as inguinal hernia repair than lower extremity surgeries, urinary retention was more commonly seen (33). Various studies reported different results related with effects of adjuvant opioid usage on urinary retention seen followed inguinal hernia repair. Gupta et al. (10) compared bupivacaine $7.5 \mathrm{mg}$ alone and $6 \mathrm{mg}$ plus fentanyl $25 \mu \mathrm{g}$ on urinary retention and couldn't find any significant differences in terms of time to urination and urethral sounding. Similarly Kallio et al (34) compared ropivacaine $15 \mathrm{mg}$ alone and ropivacaine $10 \mathrm{mg}$ plus fentanyl $20 \mu \mathrm{g}$ intrathecal in lower extremity and inguinal hernia repair operations. The authors stated no differences between two different regimens on urinary retention. Girgin et al. (22) reported shorter time for urination with levobupivacaine $5 \mathrm{mg}$ plus $25 \mu \mathrm{g}$ fentanyl than levobupivacaine $7.5 \mathrm{mg}$ alone.

Seewal et al. (35) reported similar urinary retention rates with different fentanyl doses range between 10-40 $\mu \mathrm{g}$ added bupivacaine $11 \mathrm{mg}$ intrathecal route. Also they reported that adjuvant fentanyl administration -even at high doses- didn't increased urinary retention rates. In contrast Goel et al. (11) showed increased time to urination with high dose (7.5 and $12.5 \mathrm{vs} 5 \mu \mathrm{g}$ ) intrathecal fentanyl administration in minor urological operations. In our study we found elongated duration for first urination and higher urinary retention rates in morphine administered groups compared with those in levobupivacaine only group. These results suggest that intrathecal adjuvant opioid administration is a risk factor for urinary retention.

In conclusion we achieved sufficient sensory and motor block levels with $12 \mathrm{mg}$ levobupivacaine in unilateral inguinal hernia repair. Perioperative anaesthesia and postoperative analgesia quality were higher in patients adjuvant morphine administered at $100 \mu \mathrm{g}$ dose. Although -statistically insignificant- urinary retention rates were higher in morphine added patients while patient and surgeon satisfaction rates were higher in both groups than that in levobupivacaine only group.
We can conclude that adjuvant morphine -both at 50-100 $\mu \mathrm{g}$ dosesadministration increases intraoperative and postoperative anaesthesia and analgesia quality without any significant side effects during unilateral inguinal hernia repair.

\section{Conflict of interest}

No conflict of interest was declared by the authors.

\section{REFERENCES}

1- Gristwood RW, Greaves JL. Levobupivacaine: A new safer long acting local anaesthetic agent. Exp Opin Invest Drugs 1999;8: 861-76.

2- Foster RH, Markham A. Levobupivacaine A review of its pharmacology and use as a local anesthesic. Drugs 2000; 59: 551-79.

3- Leone S, Di Cianni S, Casati A, Fanelli G. Pharmacology, toxicology, and clinical use of new long acting local anaesthetics, ropivacaine and levobupivacaine. Acta Biomed 2008; 79: 92-105.

4- Vercauteren PM, Hans G, De Decker K, Andriaensen HA. Levobupivacaine Combined with Sufentanil and Epinephrine for Intrathecal Labor Analgesia: A Comparison with Racemic Bupivacaine. Anesth Analg 2001; 93: 996-1000.

5- Alley EA, Kopacz DJ, McDonald SB, Liu SS. Hyperbaric spinal levobupivacaine: a comparison to racemic bupivacaine in volunteers. Anesth Analg 2002; 94: 188-93.

6- Glaser C, Marhofer P, Zimpfer G, Heinz MT, Sitzwohl C, Kapral S, et al. Levobupivacaine Versus Racemic Bupivacaine for Spinal Anesthesia Anesth Analg 2002; 94: 194-198.

7- Sell A, Olkkola KT, Jalonen J, Aantaa R. Minimum effective local anesthetic dose of isobaric levobupivacaine and ropivacaine administered via a spinal catheter for hip replacement surgery. $\mathrm{Br} J$ Anaesth 2005; 94: 239-42.

8- Lee YY, Muchal K, Chan CK. Levobupivacaine vs. racemic bupivacaine in spinal anaesthesia for urological surgery. Anaesth Intens Care 2003; 31: 637-41.

9- Murphy PM, Stack D, Kinirons B, Laffey JG. Optimizing the dose of intrathecal morphine in older patients undergoing hip arthroplasty. Anesth Analg 2003; 97: 1709-15.

10- Gupta A, Axelsson K, Thörn SE, Matthiessen P, Larsson LG, Holmström B et al. Low-dose bupivacaine plus fentanyl for spinal anesthesia during ambulatory inguinal herniorrhaphy: a comparison between $6 \mathrm{mg}$ and 7.5 mg of bupivacaine. Acta Anaesthesiol Scand 2003 47: 13-9.

11- Goel S, Bhardwai N, Grover VK. Intrathecal fentanyl added to intrathecal bupivacaine for day case surgery: a randomized study. Eur J Anaesthesiol 2003; 20: 294-7.

12- Korhonen AM, Valanne JV, Jokela RM, Ravaska P, Korttila K. Intrathecal hyperbaric bupivacaine $3 \mathrm{mg}+$ fentanyl 10 microg for outpatient knee arthroscopy with tourniquet. Acta Anaesthesiol Scand 2003; 47: 342-6.

13- Kalso E. Effects of intrathecal morphine, injected with bupivacaine, on pain after orthopaedic surgery. Br J Anaesth 1983; 55: 415-21.

14- Abouleish E, Rawal N, Fallon K. Combined intrathecal morphine and bupivacaine for cesarean section. Anesth Analg 1988; 67: 370-74.

15- Cole PJ, Craske DA, Wheatley RG. Efficacy and respiratory effects of lowdose spinal morphine for post-operative analgesia following knee arthroplasty. Br J Anaesth 2000; 85: 233-7.

16- Fattorini F, Ricii Z, Rocco A, Romano R, Pascarella MA, Pınto G. Levobupivacaine versus racemic bupivacaine for spinal anesthesia in orthopaedic major surgery. Minerva Anestesiol 2006; 72: 637-44.

17- Cappelleri G, Aldegheri G, Danelli G, Marchetti C, Nuzzi M, lannandrea G. Spinal anestesia with hyperbaric levobupivacaine and ropivacaine for outpatient knee arthroscopy: a prospective, randomized. Double-blind study. Anesth Analg 2005; 101: 77-82.

18- Cuvas $\mathrm{O}, \mathrm{Er} \mathrm{AE}$, Ongen E, Basar H. Spinal anesthesia for transurethral resection operations: bupivacaine versus levobupivacaine. Minerva Anestesiol 2008; 74: 697-701.

19- Luck JF, Fettes PD, Wildsmith JA Spinal anaesthesia for elective surgery: a comparison of hyperbaric solutions of racemic bupivacaine, levobupivacaine, and ropivacaine. Br J Anaesth 2008; 101(5): 705-10.

20- Casati A, Moizo E, Marchetti C, Vinciguerra F. A prospective randomized, double-blind comparison of unilateral spinal anesthesia with hyperbaric bupivacaine, ropivacaine, or levobupivacaine for inguinal herniorrhaphy. Anest Analg 2004; 99: 1387-92.

21- Demiraran Y, Yucel I, Akcali GE, Degirmenci E, Sezen G, Iskender A. Adding intrathecal morphine to unilateral spinal anesthesia results in better pain relief following knee arthroscopy. J Anesth 2008; 22: 367-72.

22- Girgin NK, Gurbet $A$, Turker G, Bulut $T$, Demir S, Kilic N, et al. The combination of low-dose levobupivacaine and fentanyl for spinal anaesthesia in ambulatory inguinal herniorrhaphy. J Int Med Res 2008 ; 36: 1287-92. 
23- Kuusniemi KS, Pihlajamäki KK, Pitkänen MT, Helenius HY, Kirvelä OA. The use of bupivacaine and fentanyl for spinal anesthesia for urologic surgery. Anesth Analg 2000; 91: 1452-6.

24- Goodarzi M, Narasimhan RR. The effect of large-dose intrathecal opioids on the autonomic nervous system. Anesth Analg 2001; 93: 456-9.

25- Slappendel R, Weber EWG, Dirksen R, Gielen MJM, van Limbeek J. Optimization of the dose of intrathecal morphine in total hip surgery: A dose-finding study. Anesth Analg 1999; 88: 822-6.

26- Rathmell JP, Pino CA, Taylor R, Partin T, Viani BA. Intrathecal morphine for postoperative analgesia: A randomized controlled dose ranging study after hip and knee arthroplasty. Anesth Analg 2003; 97: 1452-7.

27- Grattidge P. Nausea and vomiting after major arthroplasty with spinal anaesthesia including morphine: a randomised trial of subhypnotic propofol infusion as prophylaxis. Acta Anaesthesiol Scand 1998; 42: 1247.

28- Auroy Y, Narchi P, Messiah A, Litt L, Rouvier B, Samii K. Serious complications related to regional anesthesia: Results of a prospective survey in France. Anesthesiology 1997;87:479-86.

29- Kehlet H, Aasvang E. Groin hernia repair: Anesthesia W J Surg 2005; 29: 1058-61.
30- Nordin P, Zetterström H, Gunnarsson U, Nilsson E. Local, regional or general anaesthesia in groin hernia repair: multicentre randomized trial. Lancet 2003; 362: 853-8.

31- Pekka Tarkkila. Complications associated with spinal anesthesia. In, Brendan T Finucane (ed), Complications of Regional Anesthesia, 2nd ed., Springer, 2007; 149-166

32- van Veen RN, Mahabier C, Dawson I, Hop WC, Kok NF, Lange JF et al Spinal or local anesthesia in lichtenstein hernia repair: a randomized controlled trial. Ann Surg 2008; 247: 428-33.

33- Korhonnen AM. Use of spinal anesthesia in day surgery. Curr Opin Anaesthesiol 2006; 19: 612-16.

34- Kallio H, Snäll EV, Suvanto SJ, Tuomas CA, livonen MK, Pokki JP et al. Spinal hyperbaric ropivacaine-fentanyl for day-surgery. Reg Anesth Pain Med 2005; 30: 48-54

35- Seewal R, Shende D, Kashyap L, Mohan V. Effect of addition of various doses of fentanyl intrathecally to $0.5 \%$ hyperbaric bupivacaine on perioperative analgesia and subarachnoid-block characteristics in lower abdominal surgery: a dose-response study. Reg Anaesth Pain Med 2007; 32: 20-6. 